\title{
artigo
}

Martins, S.S.; Weiller, T.H.; Megier, E.R.; Gomes, B.C.F.;

Planejamento das ações desenvolvidas pelas equipes de estratégia de saúde da familia: revisão integrativa

\section{Planejamento das ações desenvolvidas pelas equipes de estratégia de saúde da família: revisão integrativa}

Planning of actions developed by family health strategy teams: integrative review

Planificación de acciones desarrolladas por los equipos de estrategia de salud familiar: revisión integrativa

\section{RESUMO}

Objetivo: identificar e analisar as evidências disponiveis na literatura acerca do processo de planejamento das ações desenvolvidas por equipes de Saúde da Familia. Método: revisão integrativa realizada em julho de 2020. Resultados: Foram selecionados 31 artigos, os quais apontaram fragilidades (gestão normativa, falta de estrutura e conhecimento para planejar) e potencialidades (cogestão com trabalhadores e comunidade, apoio matricial e institucional) Conclusão: são necessários mais estudos para aprimorar metodologias passiveis de aplicação prática e ações de educação permanente para fomentar e institucionalizar a cultura de planejamento.

DESCRITORES: Planejamento em saúde; Estratégia Saúde da Família; Sistema Único de Saúde; Enfermagem.

\section{ABSTRACT}

Objective: to identify and analyze the evidence available in the literature about the planning process of actions developed by Family Health teams. Method: integrative review carried out in July 2020. Results: Thirty-one articles were selected, which indicated weaknesses (normative management, lack of structure and knowledge to plan) and potentialities (co-management with workers and community, matrix and institutional support) Conclusion: more studies are needed to improve methodologies that can be applied in practice and permanent education actions to foster and institutionalize the planning culture.

DESCRIPTORS: Health planning; Family Health Strategy; Unified Health System; Nursing.

\section{RESUMEN}

Objetivo: identificar y analizar la evidencia disponible en la literatura sobre el proceso de planificación de las acciones desarrolladas por los equipos de Salud Familiar. Método: revisión integrativa llevada a cabo en julio de 2020. Resultados: Se seleccionaron treinta y un artículos, que indicaban debilidades (gestión normativa, falta de estructura y conocimiento a planificar) y potencialidades (co-gestión con trabajadores y apoyo comunitario, matriz e institucional): se necesitan más estudios para mejorar las metodologías que se pueden aplicar en la práctica y acciones educativas permanentes para fomentar e institucionalizar la cultura de planificación. DESCRIPTORES: Planificación de la salud; Estrategia de Salud Familiar; Sistema Único de Salud; Enfermeía.

RECEBIDO EM: 23/11/2020 APROVADO EM: 19/01/2020

\section{Sharon da Silva Martins}

Enfermeira, Especialista, Mestranda em Enfermagem pela Universidade Federal de Santa Maria, Servidora Pública do Município de Santa Maria

ORCID: 0000-0002-9608-1233

\section{Teresinha Heck Weiller}

Enfermeira, Pós Doutora em Saúde Pública, Docente do Curso de Enfermagem da Universidade Federal de Santa Maria. ORCID: 0000-0003-2531-0155

\section{Elisa Rucks Megier}

Enfermeira, Especialista, Doutoranda em Enfermagem pela Universidade Federal de Santa Maria ORCID: 0000-0003-3448-9193

\section{Bruna Cristiane Furtado Gomes}

Enfermeira, Especialista, Mestre em Enfermagem, Servidora Pública do Município de Uruguaiana ORCID: 0000-0002-4327-1973 


\section{INTRODUÇÃO}

E mbora reconhecido como prática importante, o planejamento em saúde ainda é um processo incipiente nos diversos setores e serviços. Planejar em saúde significa superar a lógica de atendimento a demandas pontuais e ao improviso, racionalizando recursos para atender às necessidades das pessoas ${ }^{(1)}$. A descentralização no Sistema Único de Saúde promoveu a gestão compartilhada e, para que a ação das três esferas de governo seja integrada e complementar, foram instituídos parâmetros para balizar o planejamento.

Nesta perspectiva, surgiu o PlanejaSUS ${ }^{(2)}$, com intuito de ofertar subsídios teóricos e metodológicos para a institucionalização do planejamento. Mesmo com esses avanços, evidencia-se a necessidade de estudos e experiências com a criação/implementação de metodologias que subsidiem o planejamento "na ponta" do sistema de saúde, ou seja, nas unidades básicas de saúde. Assim, o presente estudo objetivou analisar as evidências disponíveis na literatura acerca do processo de planejamento das ações desenvolvidas por equipes de Saúde da Família.

\section{MÉTODO}

Foi realizado estudo de revisão integrati$\mathrm{va}^{(3)}$ a partir da questão norteadora: "quais são as evidências científicas sobre como ocorre o planejamento das ações desenvolvidas pelas equipes de Saúde da Família?", localizadas em julho de 2020, nas bases de dados da Latino-Americana e do Caribe em Ciências da Saúde (LILACS) eda National Library of Medicine/ National Institutes of Health (PubMed); nos portais do Banco de Dados em Enfermagem - Bibliografia Brasileira (BDENF), Sistema Regional de Información en Línea para Revistas Científicas de América Latina, el Caribe, España y Portugal (LATINDEX) e Scientific Electronic Library Online (SciELO).

A estratégia de busca usada foi: "planejamento em saúde" AND "Estratégia Saúde da Família” OR "ESF”. Os critérios de inclusão foram: artigos primários desenvolvidos no Brasil, disponíveis na íntegra online, em qualquer idioma, sem recorte temporal, que respondam à questão norteadora do estudo e as produções repetidas foram consideradas apenas uma vez.

Foram recuperadas 1.152 produções das quais 31 compuseram o corpus do estudo (Figura 1). Estes foram lidos na íntegra por dois membros do grupo de pesquisa GEPESC e, quando houve divergência, um terceiro colaborador foi acionado. Foi elaborado um quadro sinóptico com os artigos. Após, foram extraídas as informações que respondessem à questão de pesquisa em uma ficha própria. Foram respeitados os aspectos éticos relacionados aos direitos autorais.

\section{RESULTADOS E DISCUSSÃO}

Foram recuperados 1.152 documentos, excluídos 1.077 por não atenderem aos critérios de seleção e 44 duplicados. Foram selecionados 31 artigos para a revisão, publicados em 18 periódicos diferentes, sendo 03 em inglês e 28 em português.

Quanto ao tipo de estudo, 23 pesquisas eram qualitativas, 02 quanti-qualitativas e 04 quantitativas; 03 artigos não traziam essa especificação. Verifica-se que estudos relacionados ao planejamento das ações em Saúde da Família foram publicados a partir de 2005, com maior frequência após 2011. A partir da leitura na íntegra dos estudos, emergiram duas categorias de análise: Fragilidades no processo de planejamento e
Potencialidades e caminhos no processo de planejamento(quadros 1 e 2).

A participação da comunidade se revela insuficiente, pela não compreensão de conceitos relacionados ao planejamento e à saúde como direito ${ }^{(5,6,9,11,12)}$. Usualmente, os trabalhadores não estimulam e não se envolvem no controle social ${ }^{(4,5,8,10)}$, produzindo um cuidado normativo, que exclui o usuário das reflexões e decisões.

Os estudos relacionam fragilidades no trabalho interno das equipes ${ }^{(4,13)}$, como ênfase em ações programáticas e atendimento à demanda espontânea ${ }^{(4-6,8-11,15-23)}$, além de pouco diálogo na rede de serviços e escassez de ações intersetoriais ${ }^{(4-6,17-19,21,23)}$. Os gestores, em geral, priorizam ações padronizadas do Ministério da Saúde que não consideram especificidades e perfil epidemiológico local, com foco no financiamento $(5,7,16,20,23,27,32)$. Sem efetiva descentralização de recursos e decisões ${ }^{(5,6,15,25-27)}$, acentua-se a discrepância entre oferta e demanda de serviços ${ }^{(7,16,23)}$. A integralidade e a longitudinalidade da atenção são bastante prejudicadas neste contexto.

Práticas normativas de gestão geram desmotivação de trabalhadores ${ }^{(5,25)}$, divergência de objetivos e na construção de propostas ${ }^{(7-11,16-18,23)}$, planos que dependem de relações pessoais ${ }^{(5,8,19,20)}$ e são vistos como obrigação burocrática ${ }^{(25,28)}$. A falta de sistematizar o planejamento $^{(6-10,13,19,21,25,33)}$ e a não definição do modelo de saúde ${ }^{(8,9,12,19,21)}$ diminuem a governabilidade

\section{Figura 1: Fluxograma de seleção das produções}

Estratégia de busca

"planejamento em saúde" AND ("Estratégia Saúde da Família" OR "ESF")

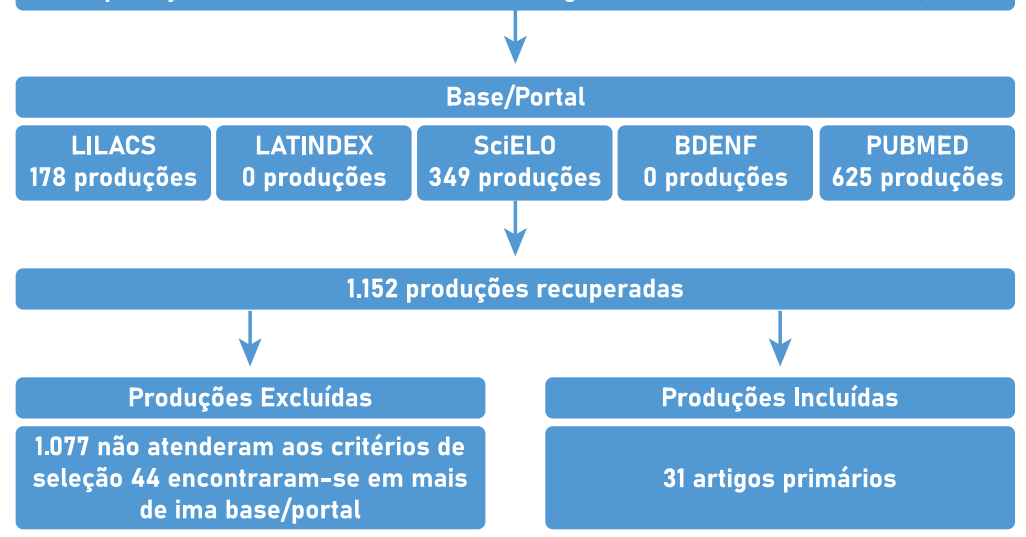

Fonte: Elaboração dos autores. 


\section{artigo}

\section{Quadro 1 - Fragilidades no processo de planejamento}

\begin{tabular}{|c|c|}
\hline \multirow{8}{*}{ 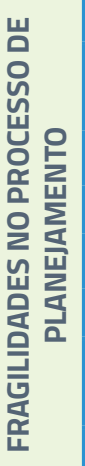 } & Pouca participação da comunidade ${ }^{(4-13)}$ \\
\hline & $\begin{array}{l}\text { Cuidado fragmentado em saúde, com ênfase em questões biológicas e sem con- } \\
\text { siderar a determinação social do processo saúde doença }{ }^{(4-6,8-11,15-23)}\end{array}$ \\
\hline & Práticas de gestão normativas prevalentes nos diversos cenários (5-11,13,15-18,23,25-28) \\
\hline & Conflito de interesses entre diferentes atores $(5,7,8,13,20,27,29)$ \\
\hline & Falta de sistematização do processo de planejamento ${ }^{(6-10,13,19,21,25,33)}$ \\
\hline & Falta de qualificação adequada para cargo de gestão $(6,7,9,11,12,16,18,19,26,27,30)$ \\
\hline & $\begin{array}{l}\text { Subutilização dos sistemas de informação por falta de conhecimento e manejo } \\
\text { inadequado dos sistemas }(4,5,10,24)\end{array}$ \\
\hline & Precarização das condições de trabalho e planejamento (7-9,15,17,19,22,26,29,30) \\
\hline
\end{tabular}

\section{Quadro 2 - Potencialidades e caminhos no processo de planejamento}

\begin{tabular}{|c|c|}
\hline \multirow{7}{*}{ 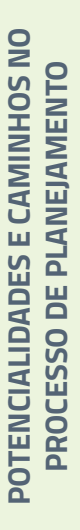 } & Cogestão e envolvimento dos membros das equipes no planejamento ${ }^{(6,7,10,13,26,28,30,32)}$ \\
\hline & Reuniões sistemáticas para discussão, reflexão e planejamento $(5,20,22,28,29,32)$ \\
\hline & $\begin{array}{l}\text { Interlocução com o apoio matricial( }{ }^{(15,20,25)} \text {, institucional }{ }^{(5,6,14,18,20,22)} \text {, Núcleo } \\
\text { Ampliado de Saúde da Família( }{ }^{(15)} \text { e instituições de ensino }{ }^{(14,26,30)}\end{array}$ \\
\hline & Integração com movimentos sociais organizados ${ }^{(10)}$ \\
\hline & $\begin{array}{l}\text { Utilização de mídias de massa como mecanismos de divulgação e compartilha- } \\
\text { mento de informações }{ }^{(4,14)}\end{array}$ \\
\hline & Instrumentalização da comunidade como protagonista e agente de mudanças ${ }^{(4,11)}$ \\
\hline & 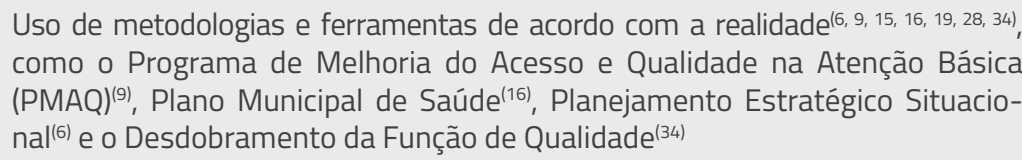 \\
\hline & acão dos autores. \\
\hline
\end{tabular}

da gestão e comprometem a implementação e consolidação de políticas públicas ${ }^{(33)}$.

Em relação às condições para planejar, os estudos apontam fragilidades técnicas, como limitações dos próprios sistemas de informação ${ }^{(4,17,19,24)}$, e dos processos de trabalho: não monitoramento e discussão de relatórios emitidos $^{(4.7,9,19,24,25)}$, falta de acesso dos trabalhadores aos relatórios e instrumentos de planejamento ${ }^{(5,12,19,26,29)}$ e ausência/incompletude da atualização dos registros ${ }^{(4,10,19,24,25)}$. Via de regra, os instrumentos de planejamento ficam restritos aos profissionais que ocupam cargos de gestão, corroborando o modelo centralizado e normativo. A insuficiência de

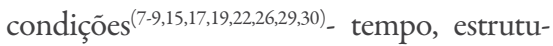
ra, sobrecarga de trabalho e muitas situações "urgentes" para resolver ${ }^{(5-11,13,15,19)}$, acentuam a precarização do planejamento. Também a falta de qualificação adequada para o cargo de gestão ${ }^{(6,7,9,11,12,16,18,19,26,27,30)}$, decorrente de critérios políticos e não técnicos ${ }^{(7,8,19,26,32)}$.
O processo de planejamento pode propiciar momentos de discussões e reflexão, fortalecer a corresponsabilização, autonomia e protagonismo dos trabalhadores. Tem potencial para mediar conflitos ao propor parâmetros técnicos para as decisões que envolvam todos os membros das equipes $(5-7,10,14,20,22,26,28,29,31)$. Foram consideradas potencialidades para o processo de planejamento o apoio matricial $^{(15,20,25)}$ e institucional ${ }^{(5,6,14,18,20,22)}$, inclusive das instituiçôes de Ensino ${ }^{(14,26,30)}$.

$\mathrm{O}$ apoio às equipes através de ações de Educação Permanente ${ }^{(47,10,14,18,25,26,30)}$ e da construção de ações conjuntas e trocas de experiências, fomenta a sistematização do planejamento, através do uso de metodologias e ferramentas ${ }^{(6,9,15,16,19,28,34)}$, como o Programa de Melhoria do Acesso e Qualidade na Atenção Básica $(\mathrm{PMAQ})^{(9)}$, Plano Municipal de Saúde ${ }^{(16)}$, Planejamento Estratégico Situacional ${ }^{(6)}$ e o Desdobramento da Função de Qualidade ${ }^{(34)}$. A ótica multidisciplinar favorece a integralidade das ações.

A construção do Planejamento com a comunidade foi possível através da integração com movimentos sociais organizados ${ }^{(10)}$, utilização de mídias de massa como mecanismos de divulgação e compartilhamento de informações ${ }^{(4,14)}$ e do estímulo ao seu protagonismo como agente de mudanças ${ }^{(4,11)}$.

\section{CONCLUSÃO}

É possível afirmar que o planejamento é uma ferramenta fundamental para transformação da realidade e melhoria da assistência em saúde. Contudo, são necessários mais estudos e experiências para aprimorar metodologias passíveis de serem aplicadas na prática cotidiana, bem como ações de educação permanente para fomentar e institucionalizar a cultura de planejamento. -

\section{REFERÊNCIAS}

1. Paim JS. Planejamento em saúde para não especialistas. In: Campos GWS, Bonfim JRA, Minayo MCS, Akerman M, Drumond Júnior M, Carvalho, YM. organizadores. Tratado de Saúde Coletiva. 2. ed. São Paulo: HUCITEC; Rio de Janeiro: FIOCRUZ, 2012. Cap. 24, p.767-782.

2. Ministério da Saúde (BR), Fundação Osvaldo Cruz. Manual de Planejamento no SUS. 1. ed. rev. Brasilia: Ministério da Saúde; 2016.
3. Paula CC, Padoin SMM; Galvão CM. Revisão integrativa como ferramenta para tomada de decisão na prática em saúde. In: Lacerda MR, Costenaro RGS, organizadoras. Metodologias da pesquisa para enfermagem e saúde: da teoria à prática. Porto Alegre: Moriá; 2018. P. 51-76.

4. Addum, FM, Serra, CG, Sessa, KS, Izoton, LM, Santos, TB. Planejamento local, Saúde Ambiental e Estratégia Saúde da Familia: uma análise do 


\section{REFERÊNCIAS}

uso de ferramentas de gestão para a redução do risco de contaminação por enteroparasitoses no município de Venda Nova do Imigrante. Physis. 2011; 21 (3), p. 955-978.

5. Andraus, SHC, Ferreira, RC, Amaral, JHL, Wernek, MAF. Organization of oral health actions in primary care from the perspective of dental managers and dentists: process of work, planning and social control. Rev. Gaúch. Odontol. 2017 dec; 65(4): p. 335-343.

6. Cubas, MR. Planejamento local: a fala do gerente de Unidade Básica de Saúde. Rev. Bras. Enferm. 2005 jun; 58(3): 278-283.

7. Ferreira, J, Celuppi, IC, Baseggio, L, Geremia, DS, Madureira, VSF, Souza, JB. Planejamento regional dos serviços de saúde: o que dizem os gestores? Saúde Soc. 2018; 27(1)

8. Gonzalez, MML. Planejamento estratégico em saúde com base em determinantes: o caso do município de Campo Bom (RS). Uma proposta metodológica para a gestão descentralizada. Ciênc. saúde coletiva. 2009 oct; 14(supl. 1): 1587-1597.

9. Pereira, LP, Nery, AA. Planejamento, gestão e ações à saúde do homem na estratégia de saúde da família. Esc. Anna Nery. 2014 dec; 18(4): 635-643.

10. Sarti, TD, Campos, CEA, Zandonade, E, Ruschi, GEC, Maciel, ELN. Avaliação das ações de planejamento em saúde empreendidas por equipes de saúde da familia. Cad. Saúde Pública. 2012 mar; 28(3): 537-548.

11. Silva, BFS, Wandekaken, KD, Dalbello-Araújo, M, Benito, GAV. A importância do planejamento como prática de gestão na microrregião de saúde de São Mateus (ES). Saúde debate. 2015 mar; 39(104):183-196.

12. Cavalcanti, YW, Lira Júnior, R, Delmondes, LN, Prado, RL, Padilha, WWN. Oral health planning in João Pessoa municipality, Paraiba State: Dental surgeons as protagonists of Basic Care. Acta sci., Health sci. 2012 jul-dec 34(2): 221-225.

13. Santos, SV, Casotti, CA, Vilela, ABA, Vieira, SNS, Nery, AA. Dificuldades e facilidades no processo de planejamento em saúde na estratégia de saúde da familia. Rev. baiana saúde pública. 2018 janmar; 42(1): 142-157.

14. Pinto LF, Rocha CMF, Mallmann CL. O uso de blogs como ferramenta de apoio à gestão em saúde no nível local. Ciênc. saúde coletiva. 2018 Out; 23 (10): 3287-3296.

15. Elia, PC, Nascimento, MC. A construção do plano local como atribuição das equipes de Saúde da Familia: a experiência de três áreas programáticas do Município do Rio de Janeiro. Physis. 2011; 21(2): 745-765.

16. Nascimento, AB, Egry, EY. Os planos municipais de saúde e as potencialidades de reconhecimento das necessidades em saúde: estudo de quatro municípios brasileiros. Saúde Soc. 2017 dec; 26(4): 861-871.

17. Pena, KS, Rollo, RM, Reuter, CLO, Santos, VCF, Riquinho, DL, Ramos, AR. Care handover to chronic conditions to regionalized planning. Rev. Gaúcha Enferm. 2020; 41(n. spe): 1-8.

18. Peruhype, RC, Mitano, F, Hoffmann, JF, Surniche, CA, Palha, PF. Planning pathways in the transfer of Directly Observed Treatment of Tuberculosis. Rev. Latino-Am. Enfermagem. 2018; 26(e3015).

19. Reuter, CLO, Maciel, PP, Santos, VCF, Riquinho, DL, Ramos, AR. Os desafios do planejamento municipal a partir da perspectiva de enfermeiras gestoras. Rev. Bras. Enferm. 2020; 73(2): 1-9.

20. Santos, AF, Machado, ATGM, Reis, CMR, Abreu, DMX, Araújo, LHL, Rodrigues, SC, et al. Institutional and matrix support and its relationship with primary healthcare. Rev. Saúde Pública. 2015 aug 11; 49(54): 1-7.

21. Sá, CR, Kuhnen, M, Santos, IF, Arruda, MP, Toassi, RFC. Planejamento em saúde bucal na atenção primária à saúde: da teoria à prática. Rev. APS. 2015 jan-mar; 18(1): 92-101.

22. Lowen, IMV, Peres, AM, Ros, CD, Poli Netto, P, Faoro, NT. Inovação na prática assistencial do enfermeiro: ampliação do acesso na atenção primária. Rev. Bras. Enferm. 2017; 70(5): 945-951.

23. Protti, ST, Silva, LMC, Palha, PF, Villa, TCS, Ruffino-Netto, $A$, Nogueira, JA, et al. A gerência da Unidade Básica de Saúde no controle da tuberculose: um campo de desafios. Rev. Esc. Enferm. USP. 2010 sept; 44(3): 665-670.

24. Duarte, MLC, Tedesco, JR, Parcianello, RR. O uso do sistema de informação na estratégia saúde da familia: percepções dos enfermeiros. Rev. Gaúcha Enferm. 2012 dec; 33(4): 111-117.

25. Cruz, MM, Souza, RBC, Torres, RMC, Abreu, DMF, Reis, AC, Gonçalves, AL. Usos do planejamento e autoavaliação nos processos de trabalho das equipes de Saúde da Familia na Atenção Básica. Saúde debate. 2014 oct; 38(n. spe): 124-139.

26. Becker, LA, Loch, MR, Reis, RS. Barreiras percebidas por diretores de saúde para tomada de decisão baseada em evidências. Rev Panam Salud Publica. 2018 mai 03; 41:1-7.

27. Cabreira, FS, Ritter, F, Aguiar, VR, Celeste, RK. Despesas municipais em atenção primária à saúde no Rio Grande do Sul, Brasil: um estudo ecológico. Cad. Saúde Pública. 2018; 34(12): 1-13.

28. Anunciação, FC, Souza, MKB. Planejamento em saúde percepções e entendimentos sobre o plano municipal de saúde. Rev. baiana saúde pública. 2011 out-dez; 35(4): 845-858.

29. Voltolini, BC, Andrade, SR, Piccoli, T, Pedebôs, LA, Andrade, V. Estratégia Saúde da Familia meetings: an indispensable tool for local planning. Texto contexto - Enferm. 2019; 28(e20170477): 1-14.

30. Ximenes Neto, FRG, Sampaio, JJC. Gerentes do território na Estratégia Saúde da Familia: análise e perfil de necessidades de qualificação. Rev. Bras. Enferm. 2007 dec; 60(6): 687-695.

31. Pinto, JM, Gerhardt, TE. Práticas avaliativas na gestão da Atenção Básica à Saúde: estudo de caso em Camaquã e Canguçu (RS). Rev. Adm. Pública. 2013 apr; 47(2), 305-326.

32. Santos, FA, Gurgel Júnior, GD, Gurgel, IGD, Pacheco, HF, Bezerra, AFB. A definição de prioridade de investimento em saúde: uma análise a partir da participação dos atores na tomada de decisão. Physis. 2015 dec; 25(4): 1079-1094.

33. Vilasboas, ALQ, Paim, JS. Práticas de planejamento e implementação de políticas no âmbito municipal. Cad. Saúde Pública. 2008 jun; 24(6): 1239-1250.

34. Volpato, LF, Meneghim, MC, Pereira, AC, Ambrosano, GMB. Planejamento da qualidade nas unidades de saúde da familia, utilizando o Desdobramento da Função Qualidade (QFD). Cad. Saúde Pública. 2010 aug; 26(8): 1561-1572 